\title{
Restricted diffusion MRI as a functional biomarker for the assessment of acute calcular upper urinary tract obstruction: initial experience
}

\author{
Heba M. Abou El Atta', Amal Abdelsattar Sakrana² and Haytham Shebel ${ }^{3^{*}}$ (i)
}

\begin{abstract}
Background: Acute renal obstruction due to stone is a prevalent scenario. The diffusion-weighted magnetic resonance imaging (DWI) of the kidneys provides a noninvasive information on renal function. Our objective is to prospectively assess the potential role of DWI to predict the signal changes of a kidney with acute calcular obstruction.

Results: Chi-square and Fissure exact tests were used to assess the association of diffusion signal changes among patients and control groups. Cohen's Kappa test was run to determine the degree of agreement between the two radiologists. An independent sample $t$-test was performed to assess the significant difference among ADC values between the two groups. Restricted signals of the obstructed kidneys showed a statistically significant difference when compared with contralateral unobstructed kidney and control group with $p$ value $(0.001)$ and $(0.01)$, respectively. Furthermore, there is a moderate agreement between the two radiologists $K=0.7, p=0.001$. There is no statistically significant difference in ADC values when comparing the obstructed kidney and the contralateral unobstructed kidney of the patient group or with the control group $p$ value (0.06) and (0.05), respectively.

Conclusion: Restricted signals of the obstructed kidney by DWI may be a helpful tool in diagnosing acute unilateral renal obstruction and can affect its management; however, it needs further validation by more studies.
\end{abstract}

Keywords: Magnetic resonance imaging, Nephrolithiasis, Diffusion MRI, Urologic diseases, Urinary calculi

\section{Key points}

- A new application of MRI diffusion in acute renal obstruction.

- Restricted signals were present in a significant number of obstructedkidneys.

- MRI can be used in diagnosis of indeterminate acute renal obstruction.

\footnotetext{
${ }^{*}$ Correspondence: haythamshebel@gmail.com

${ }^{3}$ Radiology Department, Urology and Nephrology Center, Mansoura

University, El Gomhorea St., Mansoura, Egypt

Full list of author information is available at the end of the article
}

\section{Background}

Acute obstructive uropathy is a commonly encountered condition occurring in a broad group population. One of the most prevalent causes of acute obstruction is a renal stone. Unilateral ureteral stone obstruction is affecting up to $15 \%$ of the population [1]. Usually, with this unilateral obstruction, serum creatinine is not affected unless the condition became bilateral. During obstruction, the kidneys have functional, morphological, and biochemical changes; these changes can finally lead to renal function loss. Therefore, early diagnosis and intervention can preserve the affected kidney [2,3].

The imaging gold standard to diagnose and follow upper urinary tract obstruction is antegrade perfusion 
pressure-flow test (Whitaker test) and renal diuretic scintigraphy. However, both techniques have many limitations and disadvantages, like the exposure of significant radiation with diuretic scintigraphy and its inaccurate estimation, especially in patients with excessive dilatation of the collecting system or less than $20 \%$ difference between both renal functions [4, 5]. The Whitaker test is also an invasive technique that needs a particular skill that might need high experience $[6,7]$. Furthermore, both techniques have no wide availability. Despite the Doppler ultrasonography availability, its value in renal obstruction assessment has many controversies, so its clinical implementation still has no clinical significance [8].

Computed tomography $[\mathrm{CT}]$ is the imaging modality of choice in the diagnostic workflow of urinary tract stone and essential to determine the level of intervention required. Computed tomography is superior to magnetic resonance imaging [MRI] in stone detection [9]. Also, secondary signs with CT, such as the decrease in attenuation of the renal parenchyma and obliteration of renal sinus fat, can suggest acute obstruction. Furthermore, the diagnosis of acute obstruction is based on clinical symptoms and laboratory findings, which are not always very reliable and may not be evident in every case. Even the morphological dilatation detected by $\mathrm{CT}$ does not differentiate acute from chronic renal obstruction. Additionally, this dilatation could be due to other causes rather than obstruction [10, 11].

Diffusion-weighted magnetic resonance imaging (DWI) and apparent diffusion coefficient (ADC) of the kidneys provides noninvasive information on renal function in healthy volunteers and many renal function disorders [12-14].

One of the important implications of DWI is diagnosis of acute brain infarction with high confidence based on restricted signals at the affected area. Few studies have applied the DWI sequence to evaluate obstructive uropathy of the affected kidneys [10, $15,16]$. These studies focused mainly on ADC values changes. However, to the best of our knowledge, no studies tried to assess the obstructed kidney's signal changes. Based on this knowledge, we sought to assess the signal changes on the DWI of the kidney presented by acute ureteral obstruction due to a stone where the acute backpressure effect can yield similar changes.

Therefore, the purpose of this study was to prospectively assess the potential role of DWI to predict the signal and functional changes of a kidney with acute calculus obstruction.

\section{Methods}

\section{Patient population}

This prospective cohort study was approved by the local ethics committee and written informed consent had obtained from all individuals. This study was performed in accordance with the declaration of Helsinki;1964.

The inclusion criteria included patients with the stone disease and normal renal function. The exclusion criteria were chronic kidney disease, obstructive uropathy due to causes other than stone, hepatic or splenic disorder, and renovascular disorder. One of these patients was referred he had received a second session of Extra Corporeal Shock Wave Lithotripsy [ESWL]. The standard protocol of the management was done for all of them included clinical examination, analgesic administration to relief the pain and non-contrast CT to detect a potentially urinary stone. All patients were diagnosed with ureteric stones related to the affected site of pain.

Additionally, an appropriate management plan was designed for each one of them. MRI examinations for all patients were done after receiving the appropriate renal colic management, and no contraindication to MRI examination. The mean duration of the time between the arrival to the emergency department to apply MRI examination was $30 \mathrm{~min}$ range [20 to $45 \mathrm{~min}$ ] and maximum up to $8 \mathrm{~min}$ range [5-8 $\mathrm{min}$ ] from taking analgesia before its effect could be achieved, while the mean duration between the initial symptoms and MR imaging was $13 \mathrm{~h}$ [range, 10-24 h].

Another group of sixteen healthy volunteers was involved in control group, matched to the patients regarding age and sex ( 4 women, 12 men). The control group had no history of renal disease, previous stone formation, or related renal disorders. Additionally, the control group had no history of hypertension, vascular or systemic diseases, and no recent medication history. All the control group underwent the same protocol of MRI examination.

\section{MRI protocol}

MR imaging was performed with a 1.5-T unit (Magnetom Avanto; Siemens, Germany) using a 12-channel body coil used for all sequences.

For morphologic evaluation, axial T2-weighted halfFourier rapid acquisition was performed with the following parameters (time of repetition (TR), 10,000$14,000 \mathrm{~ms}$; time to echo (TE), 80-90 ms; section thickness, $5 \mathrm{~mm}$; intersection gap, $0 \mathrm{~mm}$; matrix, $256 \cdot 160$; the number of excitations (NEX), 2; field of view (FOV), $36 \mathrm{~cm}$ ).

For functional evaluation, axial and coronal multi-section echo-planar diffusion-weighted MR imaging (DWI) 
was performed with the following parameters: (thickness, $5 \mathrm{~mm}$; intersection gap, $1 \mathrm{~mm}$ ), the field of view, $36 \mathrm{~cm}$; matrix, $192 \times 192$; bandwidth, $1446 \mathrm{~Hz}$ per pixel; and partial Fourier, 6/8. Diffusion gradient b values were applied (in seconds per square millimeter) as follows: $\mathrm{b}$ values were $(0,50,100,200,500$ and $800 \mathrm{~s} / \mathrm{mm} 2)$. These values were applied in three orthogonal planes to minimize the effects of diffusion anisotropy. Respiratory triggering was used to reduce physiological motion artifact.

\section{Image analysis}

Two experienced radiologists with 17 and 12 years in body MRI imaging [HS and HA, respectively] were involved independently in interpreting MRI sequences. Both radiologists were blinded to the results of CT. From a statistical point, we have excluded the presence of the stones as it was a constant finding in all patients. We evaluated the associations between other CT findings [hydronephrosis and perinephric strands] as absence or presence with the diffusion pattern.

Regarding DWI signals, both radiologists have evaluated both kidneys independently, using the signals of the spleen and the signal pattern of the kidneys in the control group as a reference for judgment. We compared the signal pattern of DWI at both kidneys with that of the control group. When there was a restricted bright signal of one kidney, we compared its signal with the contralateral side and the spleen. Kidneys were assigned as positive when there is a diffuse increased signal intensity of the renal parenchyma higher than that of the control group or contralateral side and near the splenic signal intensity parenchyma.

Regarding the interpretation of ADC measures, a circular region of interest (ROIs) was placed in the upper pole, mid-zone, and lower pole of the parenchyma at both kidneys to take the average ADC value of the affected kidneys as well as the control group. In the case of the control group, ADC values at both kidneys were averaged to give a single value. The measurement of ADC values was done with Syngo VB17 software.

\section{Statistical analysis}

A sample size estimation was calculated to be at least 16 patients and 16 control subjects from a power analysis based on previous diffusion-weighted MR imaging results in native kidneys (9), assuming similar standard deviations. For comparison between patients and control groups regarding the diffusion restriction pattern, nominal variables were used to assess if there is a significant association between diffusion changes at both groups using Chi-square or Fisher Exact tests. Additionally, the same tests were used to compare the association between CT signs other than stones and DWI pattern at both groups.

Cohen's Kappa test was run to determine whether there was an agreement between the two radiologists on whether there was a restricted or free diffusion of the kidneys at the patient's group compared with the control group and the contralateral kidneys of the same patients.

ADC values for both groups showed no violation of normal distribution as assessed by the Shapiro-Wilk test and no significant Skewness or kurtosis with comparable means and median at both groups. An independent sample $t$-test was performed to assess the significant difference among ADC values between the two groups. For all statistical tests, a $p$ value of less than 0.05 was considered a statistically significant difference. Statistical analyses were performed with STATA/IC Version 15.and SPSS software (SPSS, version 23, SPSS, Chicago).

\section{Results}

Forty patients referred from the urology department due to acute renal colic; however, 18 (four women, 14 men; mean age, 34 years; age range,25-54 years) patients were included in this study based on our inclusion criteria All patients presented with renal colic had ureteric stones with an average mean diameter of $3 \mathrm{~mm}$, range $(2-5 \mathrm{~mm}), 12$ cases in the distal ureter, and 6 cases in the proximal ureter.

\section{CT findings}

Perinephric strands without hydronephrosis in four cases and with hydronephrosis in 3 cases while the remaining 11 cases with no significant hydronephrosis or perinephric strands. There were associated peripheral non-obstructing small renal stones at five patients [2 stones at the right kidney and 3 stones at the left kidney] (Table 1).

Table 1 Summary of the site of the stone and CT signs

\begin{tabular}{|c|c|c|c|c|c|c|}
\hline \multicolumn{2}{|c|}{ Site of the stone } & \multicolumn{2}{|c|}{$\begin{array}{l}\text { Non-obstructing renal } \\
\text { stone }\end{array}$} & \multicolumn{3}{|l|}{ CT signs } \\
\hline Proximal & Distal & Right & Left & Strands ++ & Strands - - & No strands or hydronephrosis \\
\hline 6 & 12 & 2 & 3 & 3 & 4 & 11 \\
\hline
\end{tabular}

NB: $[++]=$ with hydronephrosis], [- $]=$ without hydronephrosis $]$ 


\section{Assessment of restricted DWI}

The normal signal intensity of the kidneys at the control group is symmetrical low signal less than the spleen on the high $b$ value images and exhibited high signals compared with the spleen on the ADC map (Fig. 1).

The first reader [HS] identified 11 cases with restricted diffusion at the kidney on the same side of the ureteric stone. The second reader [HA] identified nine cases that had restricted diffusion patterns. There was a moderate agreement between the two reader's judgments using Cohen's Kappa, $K=0.77$ [95\% CI, 0.76 to 0.79$] p=0.001$.

Nine cases presented with renal colic had restricted signal on DWI corresponding to the same side of the stone as both radiologists identified. The restricted DWI signals had emphasized in Figs. 2, 3. This restricted signal pattern yielded a statistically significant difference compared with the contralateral unobstructed kidney and the control group using Fisher Exact test with $p$ value (0.001) and (0.01), respectively (Table 2).

Additionally, there is a significant statistical association between the diffusion pattern and CT signs other than a stone with a $p$ value $(0.006)$ using the Fisher exact test.

\section{Assessment of $A D C$ values}

The mean ADC value was $2.08 \times 10-5 \mathrm{~mm} 2 / \mathrm{sec}+0.13$, $1.97 \times 1 \times 10-5 \mathrm{~mm} 2 / \mathrm{sec}+0.13$, and $1.99 \times 10-5$ $\mathrm{mm}^{2} / \mathrm{s}+0.12$ for control, obstructed and contralateral kidney of the patient groups respectively (Fig. 4).

There is no statistically significant difference in ADC values compared between the obstructed kidney and the contralateral unobstructed kidney of the patient group or with the control group using independent sample $t$-test $p$ value (0.06) and (0.05), respectively. Additionally, no statistically significant difference in ADC values between the control group and contralateral unobstructed kidney $p$ value (0.05). Table 3 Furthermore, our results did not show a statistically significant difference in ADC values with CT findings at both groups [positive CT findings versus negative CT finding] with $p$ value (0.1).

\section{Discussion}

This pilot study showed restricted diffusion signals in $50 \%$ of the cases with unilateral acute obstruction. This restriction pattern yielded significant statistical differences compared with contralateral unobstructed kidneys and control groups; $p$ values were (0.001) and (0.01), respectively. There was a substantial agreement between the two readers in detection of restricted signals of the

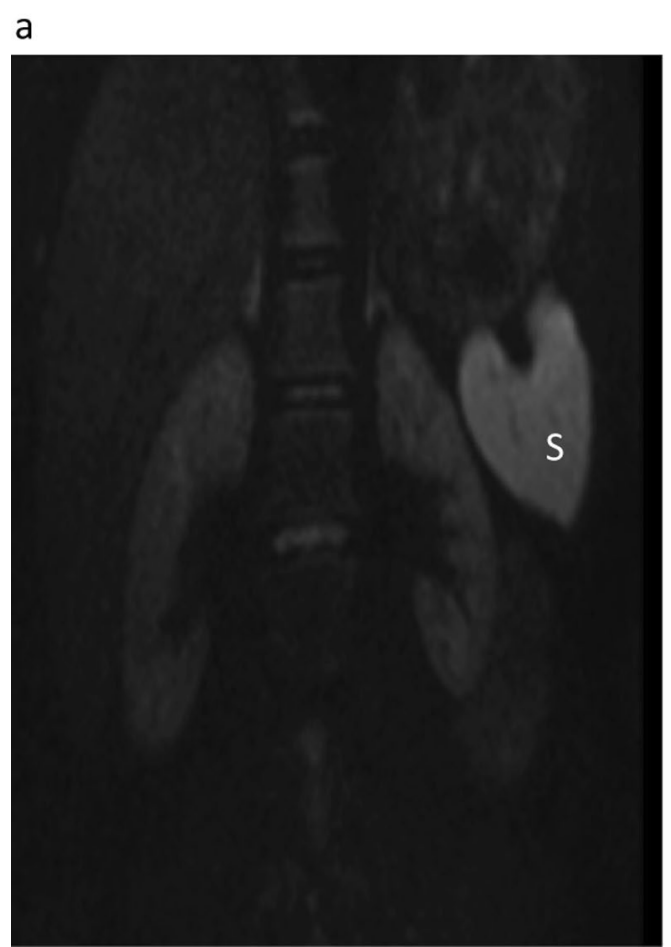

\section{b}

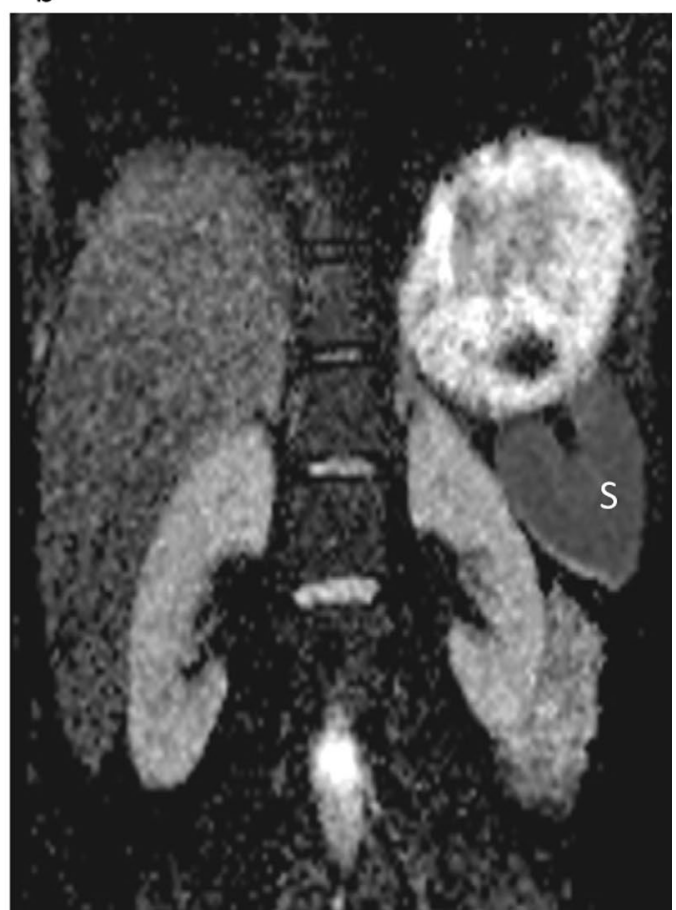

Fig. 1 Example of normal appearance of both kidneys on the diffusion-weighted image. Normal kidneys with high b value $=800$ (a), both kidneys are of low signal intensity compared with spleen as a reference. ADC map (b), both kidneys exhibit high signals when compared with the spleen, which showing low signal compared with high b value image 


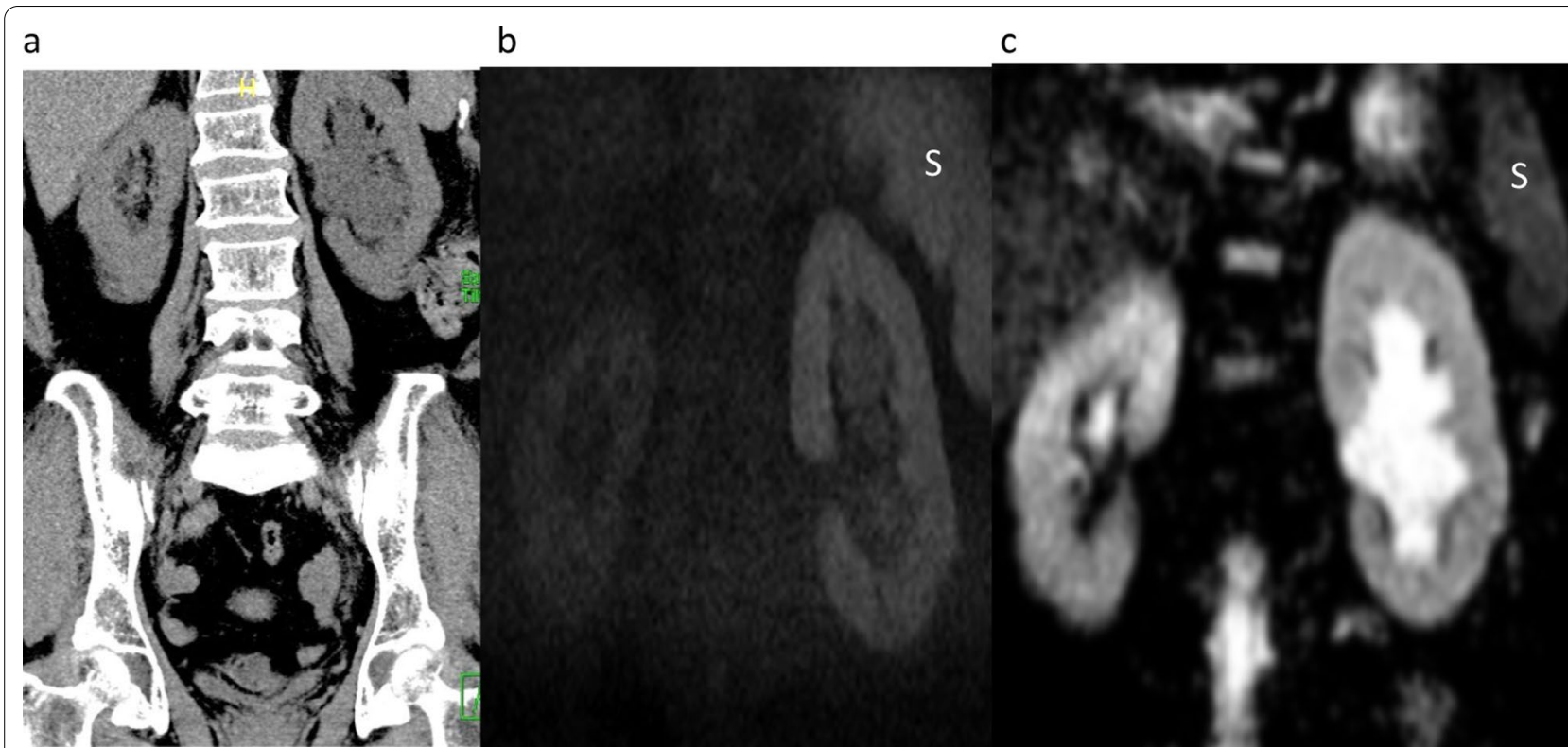

Fig. 2 A 32-year-old male patient presented with left renal colic, Coronal reformatted CT (a), shows left hydronephrosis due to stone [not shown] along the course of the left pelvic ureter, Diffusion image (b), demonstrating diffuse high signal of the left kidney compared with contralateral right kidney and almost equal to the spleen. ADC map (c) shows a reduction of the signal intensity at the left kidney with hydronephrosis compared with the right kidney and spleen. $S$ refers to spleen at both $(\mathbf{b}, \mathbf{c})$

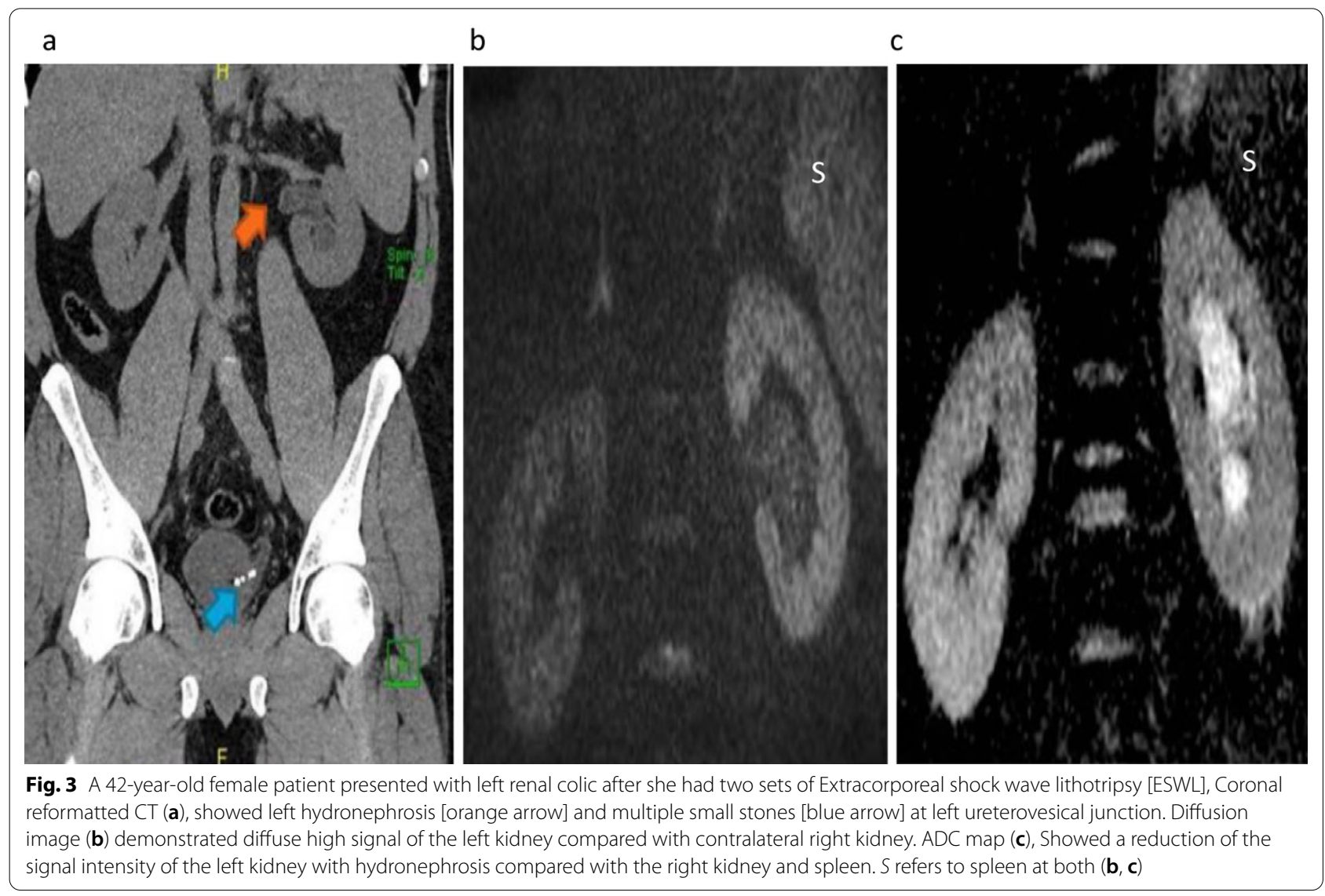


Table 2 DWI_Pattern between both groups

\begin{tabular}{|c|c|c|c|c|c|c|}
\hline & & & Group & & Total & $P$ value \\
\hline & & & Control & Patients & & \\
\hline DWI_Pattern & Free & Cases & $16_{a}$ & $9_{\mathrm{b}}$ & 25 & 0.01 \\
\hline & Restricted & Cases & $\mathrm{O}_{\mathrm{a}}$ & $9_{b}$ & 9 & 0.001 \\
\hline Total & & Cases & 16 & 18 & 34 & \\
\hline
\end{tabular}

Small subscript letter $[a, b]$ denotes there is a significant difference between both groups. Column proportions differ significantly from each other at the .05 level DWI Diffusion-weighted imaging

affected kidney, Cohen's Kappa, $K=0.77$ [ $95 \%$ CI, 0.76 to $0.79] p=0.001$. Additionally, our results showed a significant correlation between the restricted pattern and positive CT signs suggestive obstruction; $p$ value $(0.006)$. On the other hand, the ADC values in the obstructed kidneys were slightly lower when compared with the unobstructed kidneys and the control group; however, these differences were not statistically significant.
Interestingly, many authors have discussed the feasibility and reproducibility of DWI as a functional noninvasive assessment of the native and transplanted kidneys in healthy volunteers and kidney diseases [12, 17-20]. With a particular focus regarding renal obstruction, many authors have evaluated the role of ADC values [10, $15,21]$. Still, to the best of our knowledge, no one had discussed the changes in the signal pattern with renal obstruction.

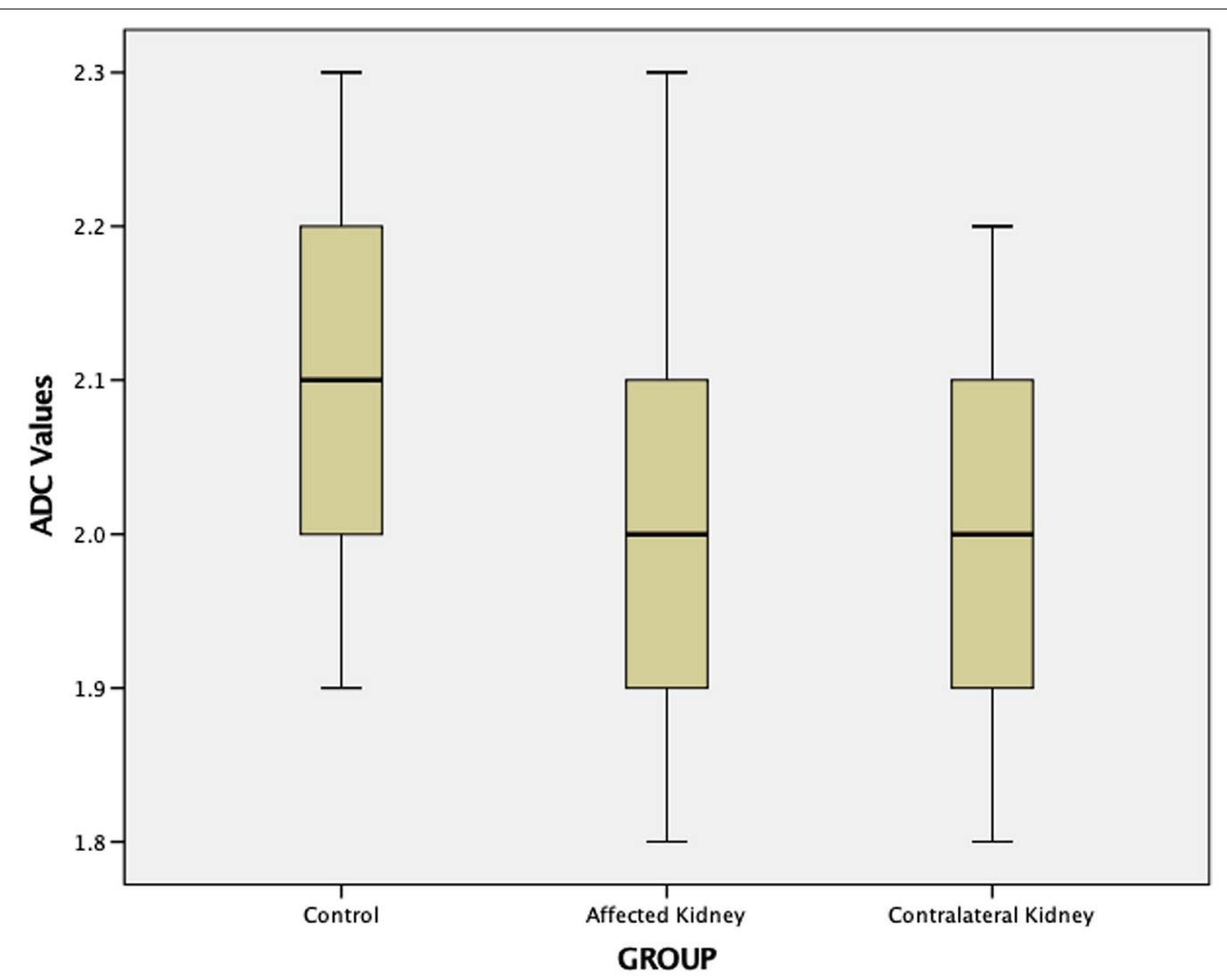

Fig. 4 Box plots of ADC (control group) ADC in, (affected kidneys) ADC in, (contralateral kidneys). For each group, the box plot indicates the mean (central horizontal line), the 75th quartile (top of box), the 25th quartile (bottom of box), there is no significant difference between ADC values of the control group and the affected kidneys, control group and contralateral kidney or between affected and contralateral kidneys of the same patient 
Table 3 Summary of ADC values and $p$ values between both groups

\begin{tabular}{lll}
\hline ADC $\left(\mathbf{1} \times \mathbf{1 0}^{-\mathbf{5}} \mathbf{~ m m}^{\mathbf{2}} \mathbf{s}\right)$ & & \\
\hline & Obstructed & Un obstructed \\
\hline Patients & $1.97+13$ & $1.99+12$ \\
$p$ value & 0.06 & \\
Control & $2.08+13$ & 0.05 \\
$p$ value* & 0.05 & \\
\hline
\end{tabular}

$p$ value $^{\sharp}$ for obstructed against unobstructed

$p$ value* control against patient groups

$A D C$ Apparent diffusion coefficient

Our results show agreement with Zulkif Bozgeyik et al. [22], who studied Twenty-six patients with acute dilatation of the pelvicalyceal system by DWI concluded no statistical significance of ADC values between the obstructed kidneys and normal kidneys in the early phase of the obstruction. Another agreement with our results with Thoeny et al. [10], used standard processing and separated diffusion and perfusion methods using high and low b values. They concluded that standard processing of diffusion showed no significant differences between ADC in the cortex and medulla of the obstructed and contralateral unobstructed kidneys. While on the same study, showed a significant difference between the obstructed and healthy kidney's ADC.

Another study, Düzenli et al. [15], examined 24 patients with unilateral ureter stones and performed DWI after $28.3 \mathrm{~h}$ as a mean duration between the initial symptoms and MRI examination. They reported a significant anatomical difference of ADC at the upper and lower poles of the obstructed kidneys compared with the same regions of the contralateral unobstructed kidneys. Moreover, another experimental study by Pedresen et al. [21] studied the effect of unilateral ureteral obstruction on the ADC in pig's kidneys. It concluded that ADC values were higher in partial chronic obstruction.

These discrepancies between ADC results in the literature are likely due to the different protocols used in these studies. Our study was concerned about detecting the early effect achieved by DWI, so we applied MRI examination as early as possible. Additionally, due to the severity of the pain, the appropriate medication was given to the patients to make them stable to perform the optimum MRI examination and standardize our results. On the other hand, some authors, as Düzenli et al. [15] preferred to examine the patients after long intervals $[13-70 \mathrm{~h}]$ to exclude the analgesic effects. This long interval allows the kidney to compensate for obstruction, concealing the expected pattern of diffusion.
The standard protocol of analgesia uses a nonsteroidal anti-inflammatory drug [NSAID], used in the current study. NSAID inhibits prostaglandin synthesis, leading to reduction of ureteric contractility and renal perfusion $[23,24]$. That is why we did not rely on the assessment of perfusion in our calculation. Additionally, that is why we were concerned about the time of examination to be as early as possible before the analgesic effect occurs.

Because of the rapid development of functional MRI sequences, many authors have tried to validate and apply these sequences to evaluate renal obstruction based on the functional changes in the kidney rather than the morphological changes. One of frequent sequences has used DWI, focusing on the quantitative component of ADC measurements; these measurements had different values and meaning of significance, as discussed before.

On the other hand, it could be time-consuming as it needs special postprocessing, mostly on a different workstation. The other qualitative component of DWI [Signal pattern] is not clearly evaluated in the literature. In the current study, we had a hypothesis based on the validated diagnosis of acute infarction of the brain [by visual determination of a restricted signal on $b$ value images with corresponding low signal on ADC images] to apply for the cases of acute calcular renal obstruction.

Furthermore, identifying the signal pattern did not need complex postprocessing or misleading calculation, making this technique likely to be more reliable and reproducible.

This study has multiple potential implications in different clinical scenarios. One of the important implications, is its value in case of indeterminate acute obstruction and avoiding inappropriate intervention or delay of management as in patients who have clinically presented with possible acute obstruction and minimal or lack of morphologic changes on ultrasound or CT, particularly kidney transplant recipients or patient with solitary kidney. Also, patients under conservative treatment for known ureteral stone developed symptoms suggestive acute on top of chronic obstruction. Furthermore, for patients with bilateral ureteral stone, selecting the priority side of intervention, if any, according to the degree of signal restriction. On the other hand, this application might potentially differentiate high from low-grade obstruction. This study had several limitations; first, we did not have a standard test as an isotope study or Whitaker test. This can be attributed to the acute clinical setting of the study, which did not allow sufficient time to perform such tests. Furthermore, these types of tests do not have wide availability and need specialized expertise. Second, we did not use separate ADC values for the medulla and cortex, which may yield a significant difference. However, this difference may not significantly provide significant power 
to be compared with the other studies due to the small sample size.

Furthermore, we looked for a simpler and more straightforward method that has not to be considered in the literature before to the best of our knowledge. Third, we did not follow the response with diffusion after the management of ureteral obstructions that would validate our hypothesis. Another possible limitation, lack of a group of patients with known ureteral stone for a longer time to differentiate between acute from chronic obstruction.

\section{Conclusions}

This is a pilot study provides a feasibility study and a positive trial of a new implementation of a known hypothesis regarding the restricted signal pattern of diffusion-weighted MRI with acute calcular unilateral obstruction. However, it needs more validation by future studies with larger sample size.

\section{Abbreviations}

ADC: Apparent diffusion coefficient; CT: Computed tomography; DWI: Diffusion-weighted magnetic resonance imaging; MRI: Magnetic resonance imaging.

\section{Acknowledgements}

None.

\section{Authors' contributions}

$\mathrm{HA}$ : Was responsible for collection of the data and interpreting MRI Images, in addition to writing the manuscript. AS: Was responsible for collecting the data interpreting CT Images in addition to writing the manuscript. HS: Was responsible for planning for study design, interpreting MRI images and performed the statistical analysis with substantial involvement in the writing and reviewing the whole manuscript. All authors read and approved the final manuscript.

\section{Funding}

None.

\section{Availability of data and materials}

The datasets used and/or analyzed during the current study are available from the corresponding author on reasonable request.

\section{Declarations}

\section{Ethics approval and consent to participate}

This prospective cohort study was approved by Institutional consent from the board of Security Forces Hospital, Kingdom Saudi Arabia. The reference number is not applicable.

written informed consent had obtained from all individuals.

\section{Consent for publication}

Written informed consent had obtained from all individuals.

\section{Competing interests}

The authors declare that they have no competing interests.

\section{Author details}

${ }^{1}$ Radiology Department, Student Hospital, Mansoura University, Mansoura, Egypt. ${ }^{2}$ Radiology Department, Mansoura University, Mansoura, Egypt.
${ }^{3}$ Radiology Department, Urology and Nephrology Center, Mansoura University, El Gomhorea St., Mansoura, Egypt.

Received: 31 July 2021 Accepted: 20 September 2021

Published online: 04 October 2021

\section{References}

1. Moe OW (2006) Kidney stones: pathophysiology and medical management. Lancet 367:333-344

2. Vaughan ED Jr, Marion D, Poppas DP, Felsen D (2004) Pathophysiology of unilateral ureteral obstruction: studies from Charlottesville to New York. J Urol 172:2563-2569

3. Klahr S (1983) Pathophysiology of obstructive nephropathy. Kidney Int 23:414-426

4. O'Reilly PH (2003) for the Consensus Committee of the Society of Radionuclides in Nephrourology: standardization of the renogram technique for investigating the dilated upper urinary tract and assessing the results of surgery. BJU Int 91:239-243

5. Dacher J, Pfister C, Thoumas D et al (1999) Shortcomings of diuresis scintigraphy in evaluating urinary obstruction: comparison with pressure flow studies. Pediatr Radiol 29:742-747

6. Veenboer PW, de Jong TP (2011) Antegrade pressure measurement as a diagnostic tool in modern pediatric urology. World J Urol 29:737-741

7. Lupton EW, George NJ (2010) The Whitaker test: 35 years on. BJU Int 105:94-100

8. Tublin ME, Bude RO, Platt JF (2003) Review. The resistive index in renal Doppler sonography: Where do we stand? AJR Am J Roentgenol 180:885-892

9. Brisbane W, Bailey MR, Sorensen MD (2016) An overview of kidney stone imaging techniques. Nat Rev Urol 13:654-662

10. Thoeny HC, Binser T, Roth B, Kessler TM, Vermathen P (2009) Noninvasive assessment of acute ureteral obstruction with diffusion-weighted MR imaging: a prospective study. Radiology 252:721-728

11. Chan JH, Tsui EY, Luk SH et al (2001) MR diffusion-weighted imaging of kidney: differentiation between hydronephrosis and pyonephrosis. Clin Imaging 25:110-113

12. $X u$ Y, Wang $X$, Jiang $X$ (2007) Relationship between the renal apparent diffusion coefficient and glomerular filtration rate: preliminary experience. J Magn Reson Imaging 26:678-768

13. Ding J, Xing Z, Jiang Z et al (2019) Evaluation of renal dysfunction using texture analysis based on DWI, BOLD, and susceptibility-weighted imaging. Eur Radiol 29:2293-2301

14. Bammer R (2003) Basic principles of diffusion-weighted imaging. Eur J Radiol 45:169-184

15. Düzenli K, Öztürk M, Yıldırım IO, Erdem G (2017) The utility of diffusionweighted imaging to assess acute renal parenchymal changes due to unilateral ureteral stone obstruction. Urolithiasis 45:401-405

16. Kalayci TO, Apaydin M, Sönmezgöz F, Çalık S, Bedel Koruyucu M (2014) Diffusion-weighted magnetic resonance imaging findings of kidneys with obstructive uropathy: differentiation between benign and malignant etiology. Sci World J. https://doi.org/10.1155/2014/980280

17. Fukuda Y, Ohashi I, Hanafusa K, Nakagawa T, Ohtani S, Annaka Y et al (2000) Anisotropic diffusion in kidney: apparent diffusion coefficient measurements for clinical use. J Magn Reson Imaging 11:156-160

18. Thoeny HC, De Keyzer F, Oyen RH, Peeters RR (2005) Diffusion-weighted MR imaging of kidneys in healthy volunteers and patients with parenchymal diseases: initial experience. Radiology 235:911-917

19. Thoeny HC, Zumstein D, Simon-Zoula S et al (2006) Functional evaluation of transplanted kidneys with diffusion-weighted and BOLD MR imaging: initial experience. Radiology 241:812-821

20. Rathod SB, Kumbhar SS, Nanivadekar A, Aman K (2015) Role of diffusionweighted MRI in acute pyelonephritis: a prospective study. Acta Radiol 56:244-249

21. Pedersen M, Wen JG, Shi Y et al (2003) The effect of unilateral ureteral obstruction on renal function in pigs measured by diffusion-weighted MRI. APMIS Suppl 109:29-34

22. Bozgeyik Z, Kocakoc E, Sonmezgoz F (2009) Diffusion-weighted MR imaging findings of kidneys in patients with early phase of obstruction. Eur J Radiol 70:138-141 
23. Frokiaer J, Nielsen AS, Knudsen L, Djurhuus JC, Pedersen EB (1993) The effect of indomethacin infusion on renal hemodynamics and on the reninangiotensin system during unilateral ureteral obstruction of the pig. J Urol 150:1557-1563

24. Lowry PS, Jerde TJ, Bjorling DE, Maskel JL, Nakada SY (2005) Obstruction alters the effect of prostaglandin E2 on ureteral contractility. J Endourol 19:183-187

\section{Publisher's Note}

Springer Nature remains neutral with regard to jurisdictional claims in published maps and institutional affiliations.

\section{Submit your manuscript to a SpringerOpen ${ }^{\odot}$ journal and benefit from:}

- Convenient online submission

- Rigorous peer review

- Open access: articles freely available online

- High visibility within the field

- Retaining the copyright to your article

Submit your next manuscript at $\boldsymbol{\nabla}$ springeropen.com 